\title{
IMMUNOHISTOCHEMISTRY DETECTION AND OXIDATIVE STRESS CAUSED BY ISOSPORA CHALCHIDIS (AMOUDI, 1989) OF CHALCIDES OCELLATUS, LIZARD
}

\author{
By \\ EHSSAN A. HASSAN \\ Department of Zoology, Faculty of Science, Suez Canal University, Ismailia, \\ Egypt (Email: ehssanmm_sci@yahoo.com)
}

\begin{abstract}
Chalcides ocellatus lizards were collected during year 2018 to be investigated for the intestinal parasitic protozoa. Protozoa infection was detected using Immunohistochemistry technique, in addition to assessment of malondialdhyde levels (MDA) and antioxidants markers in intestine and liver of infected and non infected lizards. C. ocellatus lizards were found to be infected with Isospora chalchidis, which reacted positively to immunoglobulin IgG and immunoglobulin IgM. There was an increase in MDA levels in the intestine and liver of infected lizards and a significant increase of GSH levels in liver of infected lizards $(\mathrm{P}<0.001)$. For the enzymatic antioxidants, the activity of SOD was evident, there was a significant increase in the intestine and liver of infected lizards $(\mathrm{P}<0.001)$. The activity of GST and CAT revealed a significant diminished values in intestine of infected lizards $(\mathrm{P}<0.001)$. This study emphasized the role of $I$. chalchidis in inducing oxidative stress as the increases in MDA and SOD levels and the decrease in CAT, GSH and GST levels in intestine and liver of infected C. ocellatus lizards supporting occurrence of oxidative stress. Moreover, immunohistochemistry technique is an important tool in I. chalchidis detection.
\end{abstract}

Keywords: Chalcides ocellatus, Isospora chalchidis, Immunohistochemistry, oxidative stress.

\section{Introduction}

The emergence of sophisticated defense mechanisms by parasites and pathogens were almost recognized as the major evolutionary force that interposed with physiological strategies of the hosts (Schmid-Hempel, 2011). The popularity of reptiles as pets has literally been exploding worldwide. Thus, increased efforts were dedicated to the study of reptile medicine and surgery, including pathological studies and diseases diagnosis (Oros et al, 2014).

Despite using developed molecular techniques for pathogens identification, diagnosis of pathogens in reptiles was achieved by isolation, culture or histopathology. However, immunohistochemistry allowed antigens demonstration of specific pathogens in paraffin-embedded histological sections, considered the major advantage in the possibility to directly associate a specific pathogen with histologic lesions (Ramos-Vara et al, 2008; 2011).

No doubt, the parasitic infection stimulates the immune system (Costantini and Moller,
2008) and may induce a state of oxidative stress if toxic oxidants are not sufficiently counteracted by the antioxidant system (Van de Crommenacker et al, 2010). The optimization of oxidants production during this response contributed to the development of disease and tissue injury. Oxidative stress and oxidative damage to biomolecules contributed to progression of many diseases, especially in inducement of pro-inflammatory responses activation (Halliwell and Gutteridge, 2007).

Undoubtedly, the urban environment possessed definite challenges for organisms, and those side-blotched lizards were adjusting physiologically, but not unknown what fitness costs these physiological adjustments accrues (Lucas and French, 2012).

This study aimed to detect Isopora chalchidis by direct immunochemistry techniques using $\operatorname{IgG} \&$ IgM labeled antibodies. In addition to assaying lipid peroxidation as an indicator of oxidative stress, by measuring levels of malondialdhyde (MDA). Also, antioxidant enzymes activity, such as super- 
oxide dismutase (SOD), catalase (CAT), and glutathione transferase (GST), and levels of non-enzymatic antioxidant, reduced glutathione (GSH) are estimated as indicators of antioxidant defences to realize the possible variation in oxidative stress occurred in lizards during infection.

\section{Materials and Methods}

Lizard collection: This study was achieved in Abu Rawash (Giza Governorate) about 16 $\mathrm{km}$ west of Cairo City, The Capital and 8 km North-west of Giza Pyramids. Thirty $C$. ocellatus lizards were examined during year 2018. The lizards were transported alive to the laboratory and euthanized. Intestine of every lizard removed and examined for presence of cocccidian parasites. Recovered protozoa were identified by generic morphological characters using light microscopy (Amoudi, 1989; El Toukhy et al, 1996).

Histological used techniques and Immunohistochemistry (Direct method): Small pieces of intestine of both infected and noninfected pigeons were fixed in Bouin's solution for $48 \mathrm{hr}$; post fixed in several changes of $70 \%$ alcohol. Specimens then dehydrated in ascending series of alcohol $(70 \%, 80 \%$, $90 \%, 100 \%$ \& $100 \%$ ); cleared in two changes of xylene and infiltrated with paraffin wax, then paraffin blocks of specimens were made. Histological sections were stained by Harris' haematoxylin and counterstained by Eosin (Humason, 1979).

As to immunohistochemistry detection technique, the primary antibody was conjugated directly to the label, as one step staining method where a labeled antibody was involved. There was a direct reaction between IgG \& IgM the antigens in tissue sections, where the antibody employed for labeling formalin-fixed, paraffin-embedded and sections. Pre-treatment of tissues were carried out using EnVision FLEX Target Retrieval Solution, High pH (50X) (Code K8004). Pre-programming of staining steps and incubation times were achieved by software of Dako Autostainer/Autostainer Plus instruments, using the following protocols:
Counterstaining in hematoxylin was recommended by using EnVision FLEX (Code K 8018).

Oxidative stress markers determination: Prior to dissection, Intestine and liver of infected and non-infected lizards were perfused with a phosphate buffered saline solution (PBS), $\mathrm{pH} 7.4$ containing $0.16 \mathrm{mg} / \mathrm{ml}$ heparin, then homogenized in $5-10 \mathrm{ml}$ cold buffer $(100 \mathrm{~mm}$ potassium phosphate, $\mathrm{pH}$ 7.0, containing 2mm EDTA)/ gram tissue. After that, centrifugation was carried out at $4,000 \mathrm{rpm}$ for 15 minutes at $4^{\circ} \mathrm{C}$ and the supernatant was collected. Then, $0.5 \mathrm{ml}$ of icecold extraction reagent was added to $1 \mathrm{ml}$ of supernatant in glass test tube and vortex for at least 30 seconds. After Centrifugation at $4000 \mathrm{rpm}$ at $20^{\circ} \mathrm{C}$ for 10 minutes, the aqueous upper layer was collected in two dry tubes, the first tube for enzymatic antioxidant parameter to immediate assay and the second tube for non-enzymatic antioxidant and stored at freeze and kept at $-20^{\circ} \mathrm{C}$.

The supernatant obtained after centrifugation of liver and ileum homogenates was used for the determination of MDA levels (n.mol/g) (Satoh, 1978), GSH (mg/dl) levels (Beutler et al, 1963) and enzymatic antioxidants activities, CAT (u/g) (Aebi, 1984), GST (u/g) (Habig et al, 1974), and SOD $(\mathrm{u} / \mathrm{g})$ (Nishikimi et al, 1972) were colorimetrically determined in tissue homogenate.

Statistical analysis: Unpaired t- test was used for studying the significant differences between groups. All data were analyzed with the software packages Microsoft SPSS version 20, for statistical evaluation. Value of $P$ $<0.001$ reflected levels of significance.

\section{Results}

Endogenous stages and immunohistochemistry detection: Histological examination revealed presence of $I$. chalchidis endogenous stages in the intestine epithelium. Histological sections revealed the presence of uninucleated meronts which are oval measuring about (7.1-10.7) $\mu \mathrm{m}$, Binucleated

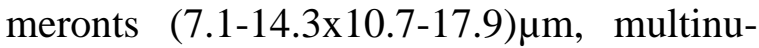
cleated meronts (7.1-10.7 $\times 21.4-24.9) \mu \mathrm{m}$ 
with merozoites (Fig. 1A;B). Young macrogamonts (10.7-14.3x14.3-17.9) $\mu \mathrm{m}$ were ovoid to spherical, have a prominent large nucleus (Fig. 1C; E). Immature microgamonts devoid of a distinct nucleolus (17.9$25 \times 35.7-49.9) \mu \mathrm{m}$ recognized by a large number of small nuclei, arranged at the periphery of their cytoplasm with consolidating wall forming body (WFB) (Fig. 1D;E), Zygote with peripheral (WFB1), and central (WFB2) wall forming bodies (Fig. 1H), Young developing oocysts (Fig.1G) and unsporulated oocyst (18.4-21.4x21.4-32.1) $\mu \mathrm{m}$ (Fig. 1I).

As immunoreactivity of $I$. chalchidis, it reacted positively to $\mathrm{IgG}$ (Fig. 2A;B) and IgM (Fig. 2C;D) with reaction more evident using labeled IgM.

Oxidative stress: To evaluate the host response induced by $I$. chalchidis, different parameters of oxidative stress in intestine and liver of infected lizards were assessed. First was to compare levels of the MDA and GSH in the liver and intestine tissues from non-infected and infected lizards. There was increase in MDA levels in intestine and liver of infected lizards $(11.52 \pm 0.19 ; 10.02 \pm 0.18)$ compared to non-infected ones $(8.76 \pm 0.14$; $9.36 \pm 0.14$ ), respectively. Concerning GSH, there was a significant increase in GSH levels in liver of infected lizards $(5.67 \pm 0.12)$ ( $P$ $<0.001)$ and a decreased level in the intestine $(2.04 \pm 0.03)$.

For enzymatic antioxidants, there was a significant increase in SOD activity in the intestine and liver of infected lizards (42.28 $\pm 0.13 ; 38.60 \pm 0.10)$, respectively, ( $\mathrm{P}$ $<0.001)$. Activity of GST \& CAT showed a significant diminish in intestine of infected lizards $(60.14 \pm 0.061 .08 \pm 0.09)$, respectively $\&$ in liver GST $(55.32 \pm 0.12)(\mathrm{P}<0.001)$.

\section{Discussion}

Diagnosis of a number of important diseases in humans and domestic animals had been verified by immunohistochemistry as an effective tool for precise diagnosis (Jensen et al, 1996). In this study, I. chalchidis reacted positively to IgG and IgM. Lesions associated with intranuclear coccidiosis induce oxidative stress which related to the inflammatory response activation that may be triggered by pathogenic agent and often perpetuated as a result of increased production of reactive species and consequent oxidative damage to biomolecules (Di Penta et al, 2013).

Measurement of malondialdehyde (MDA) concentrations for determination of lipid peroxidation intensity was among the commonest used methods for determination of the oxidative stress. The increase of MDA concentrations in plasma was a marker of lipid peroxidation (Moor and Roberts, 1998). As GSH and CAT are involved in the conversion of radicals into less effective metabolites, leading to increase of MDA concentrations (Kizil and Yuce, 2009)

The intracellular stages of the coccidian parasites have a crucial role in modifying their host cells widely and interfering with many host signaling pathways. In the present study, MDA activity was enhanced and the non-enzymatic antioxidant capacity was decreased showing oxidative stress.

Studies hypothesized that the decrease in CAT and GSH activity in protozoa infection might be due to the cellular defence systems that affected by excessive formation of reactive compounds and that is expressive of oxidative stress (Çam et al, 2008). Also, Kizil and Yuce (2009) noticed a significantly depressed in the GSH concentrations and CAT activities in the infected group compared to the control group in coccidiosis. Dakhil et al. (2012) reported increased level of MDA and NO resulted from stressful conditions following protozoa infection, and suggested that this increase resulted from serious inflammatory response due to oxidative damage caused by coccidian infection.

\section{Conclusion}

The study emphasized the role of I. chalchidis in inducing oxidative stress as the increases in MDA and SOD levels and the decrease in CAT, GSH and GST levels in intestine and liver of infected $C$. ocellatus liz- 
ards supporting occurrence of the oxidative stress. Immunohistochemistry proved to be an important tool in I. chalchidis detection.

\section{References}

Aebi, H, 1984: Catalase in Vitro. Meth. Enzym. 105:121-6.

Amoudi, MA, 1989: Two new species of Isospora from the desert skink (Chalcides ocellatus) from the Egyptian Desert. J. Protozool. 36: 237-8.

Beutler, E, Duron, O, Kelly, BM, 1963: Improved method for the determination of blood glutathione. J. Lab. Clin. Med. 61:882-90.

Çam, Y, Atasever, A, Eraslan, G, Kibar, M, Atalay, O, et al, 2008: Eimeriastiedae: experimental infection in rabbits and the effect of treatment with toltrazuril and ivermectin. Exp. Parasitol. 119:164-72.

Costantini, D, Moller, AP, 2009: Comp. Biochem. Phys. Part A. 153:339-44.

Dakhil, MA, Abdel-Maksoud, MA, Al-Quraishy, S, Abdel-Baki, AS, Wunderlich, F, 2012:

Gene expression in rabbit appendices infected with Eimeria coecicola. Vet. Parasitol. 186:2228.

Di Penta, A, Moreno, B, Reix, S, FernandezDiez, B, Villanueva, M, et al, 2013: Oxidative stress and proinflammatory cytokines contribute to demyelination and axonal damage in a cerebellar culture model of neuroinflammation. PLoS One. 8, 2:e54722

El-Toukhy, AA; Abdel Aziz, A, Abdel Gawad, MA, 1996: Light and electron microscopic studies of Isospora chalchidis (Amoudi, 1989) infecting Chalcides ocellatus in Egypt. J. Egypt. Soc. Parasitol_.26, 1:79-91.

Habig, WH, Pabst, MJ, Jakoby, WB, 1974: Glutathione S-transferases: The first enzymatic step in mercapturic acid formation. J. Biol. Chem. 249:7130-9.

Halliwell, B, 2007: Biochemistry of oxidative stress.Biochem. Soc. Trans. 35:1147-50.

Halliwell, B, Gutteridge, JMC, 2007: Free Padicals in Biology and Medicine. 4th ed. Oxford: Oxford University Press.

Humason, GL, 1979: Animal Tissue Techniques, $4^{\text {th }}$ Ed. Freeman and Company, San Franciso, USA.
Jensen, HE, Schønheyder, HC, Hotchi, M, Kaufman, L, 1996: Diagnosis of systemic mycoses by specific immunohistochemical tests. APMIS. 104:241-58.

Kizil, O, Yuce, A, 2009: Oxidative stress in dogs with coccidiosis. Rev. Méd. Vét. 160, 11: 495-9.

Lucas, LD, French, SS, 2012: Stress-induced tradeoffs in a free-living lizard across a variable landscape: Consequences for individuals and populations. Published online 2012 Nov 20. doi: 10.1371/journal.pone.0049895

Moore K, Roberts LC, 1998: Measurement of lipid peroxidation. Free Radic. Res. 28:659-71.

Nishikimi, M, Rao, NA, Yagi, K, 1972: Occurrence of superoxide anion in the reaction of reduced phenazine methosulphate and molecular oxygen. Biochem. Biophys. Res. Commun. 46: 849-64.

Orós, J, Arencibia, A, Jensen, HE, 2014: Immunohistochemistry as a tool for the accurate diagnosis of diseases in reptiles. In: Microscopy: Advances in Scientific Research and Education. (A. Méndez-Vilas, Ed.). V.1 Spanien: Formatex. Ramos-Vara, JA, 2011: Principles and methods in immunohistochemistry. Methods Mol. Biol. 691:83-96.

Ramos-Vara, JA, Kiupel, M, Baszler, T, Bliven, L, Brodersen, B, et al, 2008: Suggested guidelines for immunohistochemical techniques in veterinary diagnostic laboratories. J. Vet. Diagn. Invest. 20:393-413.

Satoh, K, 1978: Serum lipid peroxide in cerebro-vascular disorders determined by a new colorimetric method. Clin. Chim. Acta 90:37-43.

Schmid-Hempel, P, 2011: Evolutionary Parasitology. The Integrated Study of Infections, Immunology, Ecology, and Genetics New York: Oxford University Press.

Sies, H, 1997: Oxidative stress: Oxidants and antioxidants. Exp. Physiol. 82:291-5.

Van de Crommenacker, J, Horrocks, N, Versteegh, M, Komdeur, J, Tieleman, B, et al, 2010: Effects of immune supplementation and immune challenge on oxidative status and physiology in a model bird: implications for ecologists. J. Exp. Biol. 213, 20:3527-35.

\section{Explanation of figures}

Fig.1: Endogenous stages of I. chalchidis in the intestine of C. ocellatus (haematoxylin and eosin) (x400). A: uninucleated meront (Me1), binucleated meront(Me2), and multi nucleated meront (Me3). B: Segmented meront with developing merozoites (Me). C: developing macrogamont (Ma). D: Microgamont with peripherally arranged nuclei and WFB. E:Microgamont with nuclei of microgamets (Mi) and WFB and mature macrogamont (Ma) .F: Developing zygotes with WFB1 and WFB2 (Zy). G\&H: Zygote with HCN and WFB1. I: unsporulated 
oocyst with polar body (Oo). (IN: inclusion; WFB: consolidating wall forming body; WFB1:peripheral wall forming bodies; WFB2: Central wall forming bodies; HCN:Host cell nucleus; P:polar body).

Fig. 2: Immunoreactivity of $I$. chalchidis in intestine of $C$. ocellatus (x400). A\&B: Immnoglobulin IgG labeled for I. chalchidis antigens. C\&D: Immnoglobulin IgM labeled for I. chalchidis antigens.

Fig. 3: Levels of MDA (n.mol/g), SOD (u/g), GSH (mg/dl), GST (u/g), and CAT (u/g) in intestine of non- infected and infected lizard.

Fig. 4: Levels of MDA (n.mol/g), SOD (u/g), GSH (mg/dl), GST (u/g), and CAT (u/g) in liver of non- infected and infected lizard.

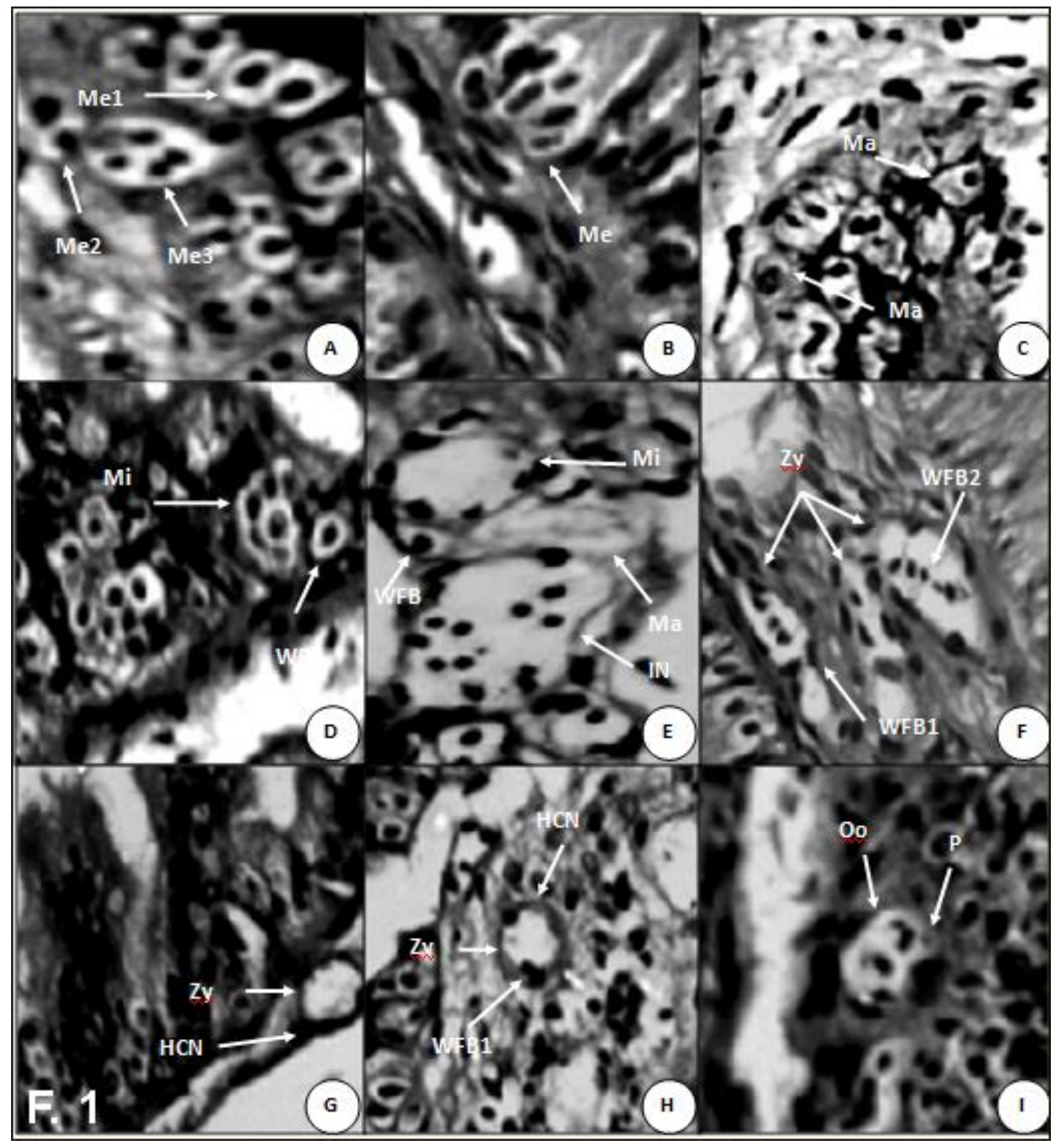



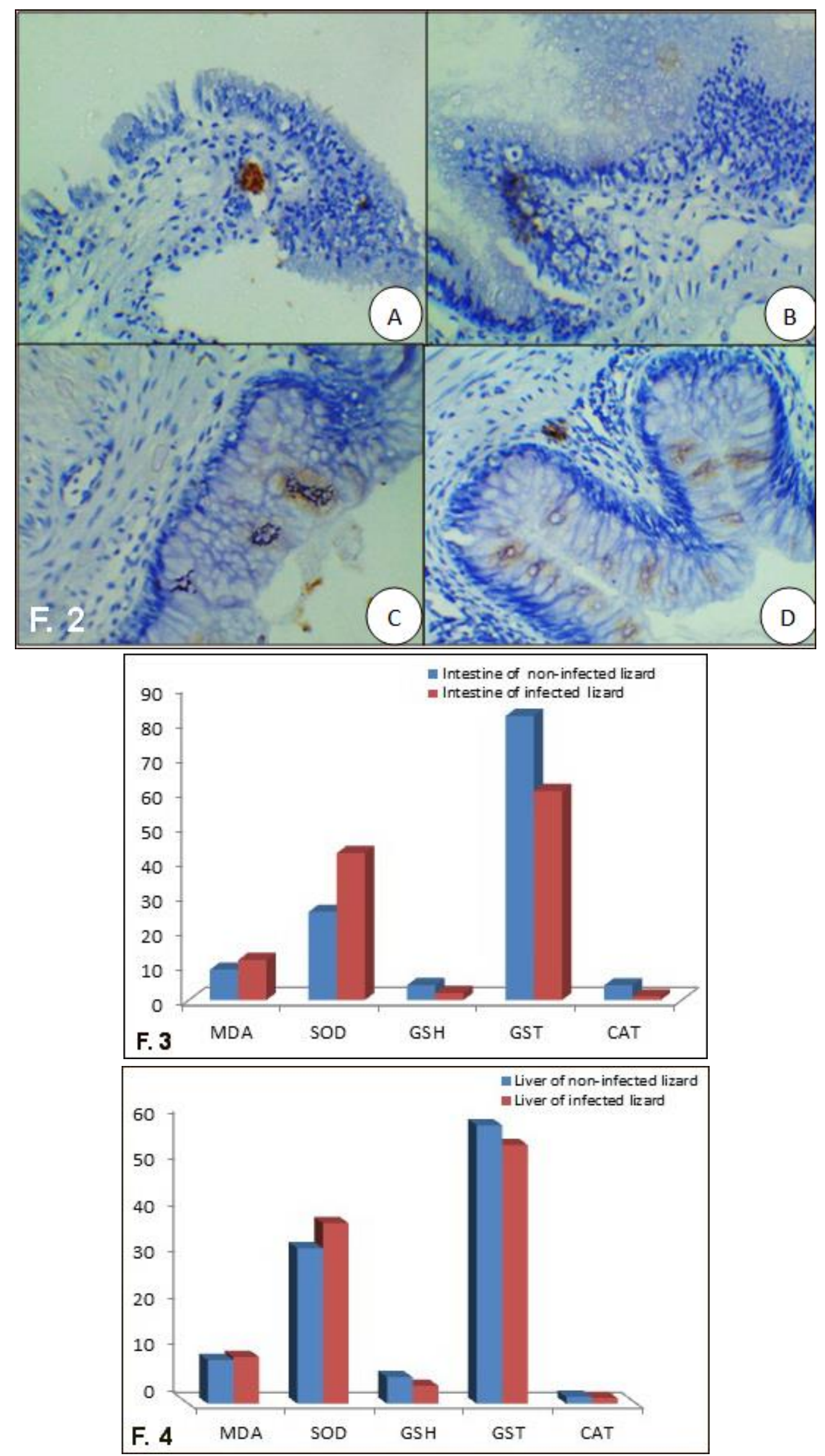